\title{
ine \\ Spoon-like Beams Generated with Exponential Phases
}

\author{
Lei Guo ${ }^{1,+}$, Tian Xia ${ }^{2, \dagger}$, Yiping Xu ${ }^{1}$, Yan Xiong ${ }^{1}$, Xian Leng ${ }^{3}$, Shaohua Tao ${ }^{2}$, Yonghong Tian ${ }^{1, *}$ \\ and Shubo Cheng $1, *(\mathbb{B}$
}

1 School of Physics and Optoelectronic Engineering, Yangtze University, Jingzhou 434023, China; 201906010@yangtzeu.edu.cn (L.G.); ypxu@yangtzeu.edu.cn (Y.X.); yxiong@yangtzeu.edu.cn (Y.X.)

2 School of Physics and Electronics, Central South University, Changsha 410083, China; tianxia@csu.edu.cn (T.X.); eshtao@csu.edu.cn (S.T.)

3 School of Packing and Materials Engineering, Hunan University of Technology, Zhuzhou 412007, China; wangchunhua@hut.edu.cn

* Correspondence: yhtian@yangtzeu.edu.cn (Y.T.); shubocheng@yangtzeu.edu.cn (S.C.)

+ These authors contributed equally to this work.

Citation: Guo, L.; Xia, T.; Xu, Y.; Xiong, Y.; Leng, X.; Tao, S.; Tian, Y.; Cheng, S. Spoon-like Beams Generated with Exponential Phases. Coatings 2022, 12, 322. https:// doi.org/ 10.3390/coatings12030322

Academic Editor: Angela De Bonis

Received: 31 December 2021

Accepted: 15 February 2022

Published: 1 March 2022

Publisher's Note: MDPI stays neutral with regard to jurisdictional claims in published maps and institutional affiliations.

Copyright: (C) 2022 by the authors. Licensee MDPI, Basel, Switzerland. This article is an open access article distributed under the terms and conditions of the Creative Commons Attribution (CC BY) license (https:// creativecommons.org/licenses/by/ $4.0 /)$.

\begin{abstract}
In this paper, we report a new kind of beam, named "spoon-like" beams, generated with the exponential phase. The intensity distributions and transverse energy flow of the spoon-like beam at the focal plane are analyzed theoretically and experimentally. The results demonstrate that the size of the spoon-like beam becomes enlarged with the increasing power exponent $n$, and the length of the spoon-like intensity trajectory becomes shorter with the increasing parameter $p$. Furthermore, there is an intensity gradient along the spoon-like trajectory of the beam, which introduces the intensitygradient force exerted onto microparticles. The experiment on optical tweezers demonstrates that the focused beams can create spoon-like traps for the two-dimensional manipulation of particles.
\end{abstract}

Keywords: exponential phases; diffraction property; optical guiding

\section{Introduction}

The use of highly focused Gaussian beams for optical trapping has been very successful [1,2]. However, a tightly focused Gaussian beam rapidly diverges away from a focus, and the use of the Gaussian beam in optical guiding and sorting is limited. In recent years, several novel beams have been proposed. LG beams, i.e., optical vortices (OVs) with the azimuthal index $l=1$, characterized by helical wavefronts and dark cores, have a well-defined orbital angular momentum (OAM) per photon. LG beams can be extensively used in the field of optical rotation for phase-gradient force exerted on particles, and optical information encoding for the intrinsic and extrinsic nature of OAM [3]. Furthermore, the optical binding of low-index and absorbing particles has also been realized by using OVs [4-6]. Noncanonical OVs, i.e., fractional OVs, radially polarized fractional vortex beams, and power-exponent-phase vortices, have been presented, and offer new ways of optical manipulation [7-12]. Bessel beams possess the ability to propagate 'diffractionfree' in free space [13-15]. The self-reconstruction property of fractional Bessel beams has been demonstrated by Tao et al. [16], and higher-order Bessel beams have azimuthal and radial-phase dependence [17]. Alonzo performed helical and conical phase fronts, and proposed a new kind of beam, the helico-conical beam, generated by multiplying the helical and conical phase functions. The beam revealed the spiral intensity distribution along the optics axis, and can potentially be used in the high surface area systems of colloids and nanoparticles [18-21]. In order to obtain the controllable intensity distributions and rotation of the beams, Nathaniel Hermosa et al. modified the helical phase by boring a hole at the center of the phase profile [22]. The intensity patterns greatly depend on the size of the bored hole. Moreover, Zhao et al. were the first to propose a new kind of power-exponentphase vortices, different from the canonical optical vortices and power-exponent-phase vortices $[23,24]$. Furthermore, we have since realized adjustable openings by adjusting the 
azimuthal or radial power index. Modified helico-conical beams have been applied for the two-dimensional manipulation of microparticles $[25,26]$. Generally, the phase profiles of the above-mentioned beams are dependent on the azimuthal or radial power index. The phase profiles dependent on the exponent function of the azimuth have not been reported. In this paper, an exponential beam with a spoon-like intensity distribution is proposed. The two-dimensional intensity patterns of the exponential beam at the focal plane are analyzed theoretically and experimentally. The far-field mapping and the transverse energy flow of the beam are also introduced to analyze the distribution of the focal field. The intensity distributions of the spoon-like beams, customized with the tailored exponential phase, are also analyzed. Optical tweezer experiments with the beam are implemented to verify the ability of the beam to manipulate microparticles.

\section{Spoon-like Beams Generated with Exponential Phases}

The phase function $\psi(r, \theta)$ of the exponential phase plate can be obtained in Equation (1):

$$
\psi(r, \theta)=l \cdot\left(\frac{\exp (\theta)}{\theta}\right)^{n} \cdot r / r_{0}
$$

where $l$ is the topological charge, $n$ is the power index ranging from 0 to $1, r_{0}$ is a normalization factor of the radial coordinate $r$, and $\theta$ is the azimuth angle which is greater than 0 and less than or equal to $2 \pi$.

In this paper, we analyze the two-dimensional intensity distribution of the exponential beam at the focal plane of a Fourier transforming lens illuminated by a plane wave encoded with a phase profile, as shown in Equation (1). In the simulations, the phase profile was sampled with a grid of $1080 \times 1080$ pixels with a pixel area of $8 \times 8 \mu \mathrm{m}^{2}$. The phase profiles calculated by Equation (1) with $l=-5$ and $n=0.4,0.5,0.6$ are shown in Figure 1a-c, respectively. The encoded phases were wrapped from 0 to $2 \pi$. Figure $1 \mathrm{~d}-\mathrm{f}$ show the focal fields of the exponential beams when $n=0.4,0.5$, and 0.6 , respectively. It can be seen that the intensity trajectory shown in Figure 1 resembles a spoon. Thus, we named this kind of exponential beam as a "spoon-like" beam. The size of the generated spoon-like beam becomes enlarged with the increasing power exponent $n$. Furthermore, the size of the spoon-like beams can be also adjusted by changing the corresponding topological charge $l$.
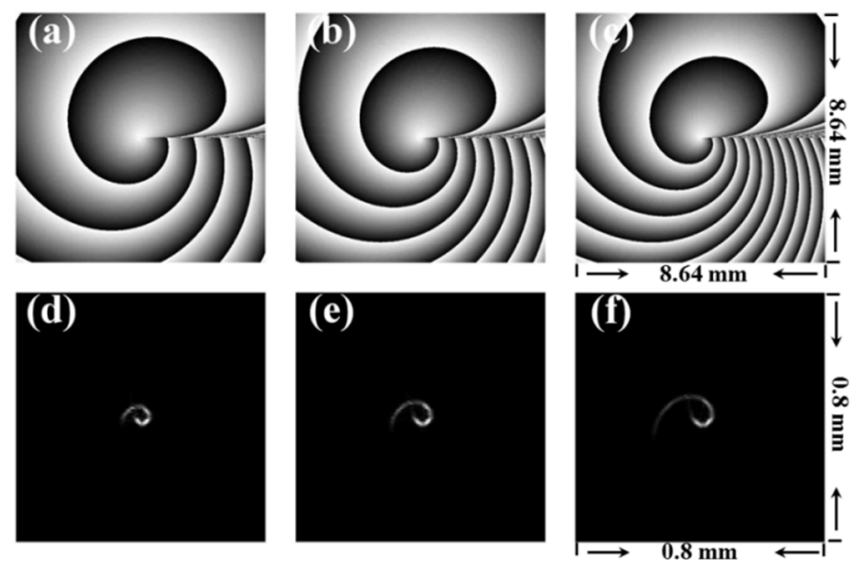

Figure 1. (a-c) The phase profiles encoded with Equation (1) with $l=-5$ and $n=0.4,0.5,0.6$, respectively. $(\mathbf{d}-\mathbf{f})$ The focal fields of the generated exponential beams with the phase profiles shown in $(\mathbf{a}-\mathbf{c})$, respectively.

A formal analysis was implemented by using the concept of local spatial frequency [27]. Local spatial frequency analysis possibly provides a basis for the observed pattern. For an object field, an approximate mapping can be provided by the local spatial frequencies defined as:

$$
\xi^{\prime}=\frac{1}{2 \pi} \frac{\partial}{\partial x} \bar{\psi}(x, y) \quad \text { and } \quad \zeta^{\prime}=\frac{1}{2 \pi} \frac{\partial}{\partial y} \bar{\psi}(x, y)
$$


Equation (2) can be arrived at by solving the Fourier integral by the method of stationary phase [28]. In order to produce a conformal mapping of the wave function, we transform Cartesian to log-polar coordinates and get the approximate mapping in polar coordinates shown in Equation (3) [29].

$$
\begin{aligned}
& \xi^{\prime}=\frac{l}{2 \pi r_{0}}\left[\left(\frac{\exp (\theta)}{\theta}\right)^{n} \cdot \cos \theta-n \cdot\left(\frac{\exp (\theta)}{\theta}\right)^{n} \cdot\left(1-\frac{1}{\theta}\right) \sin \theta\right] \\
& \text { and } \\
& \zeta^{\prime}=\frac{l}{2 \pi r_{0}}\left[\left(\frac{\exp (\theta)}{\theta}\right)^{n} \cdot \sin \theta+n \cdot\left(\frac{\exp (\theta)}{\theta}\right)^{n} \cdot\left(1-\frac{1}{\theta}\right) \cos \theta\right]
\end{aligned}
$$

For example, according to Equation (3), the frequency mapping $\left(\xi^{\prime}, \zeta^{\prime}\right)$ with $l=-5$, $n=0.4,0.5$ and 0.6 can be plotted as a spot diagram, as shown in Figure $2 \mathrm{a}-\mathrm{c}$, respectively. In the figures, the density of points is representative of intensity at the focal plane, and some points are accumulated in a spoon-like trajectory. The densities of the points shown in Figure $2 \mathrm{a}-\mathrm{c}$ are consistent with the shapes of the corresponding focal fields of the generated spoon-like beams shown in Figure 1d-f, respectively. The local spatial frequency analysis can explain the observed intensity pattern. The simulations demonstrate that the size of the beam is dependent on the power order $n$.
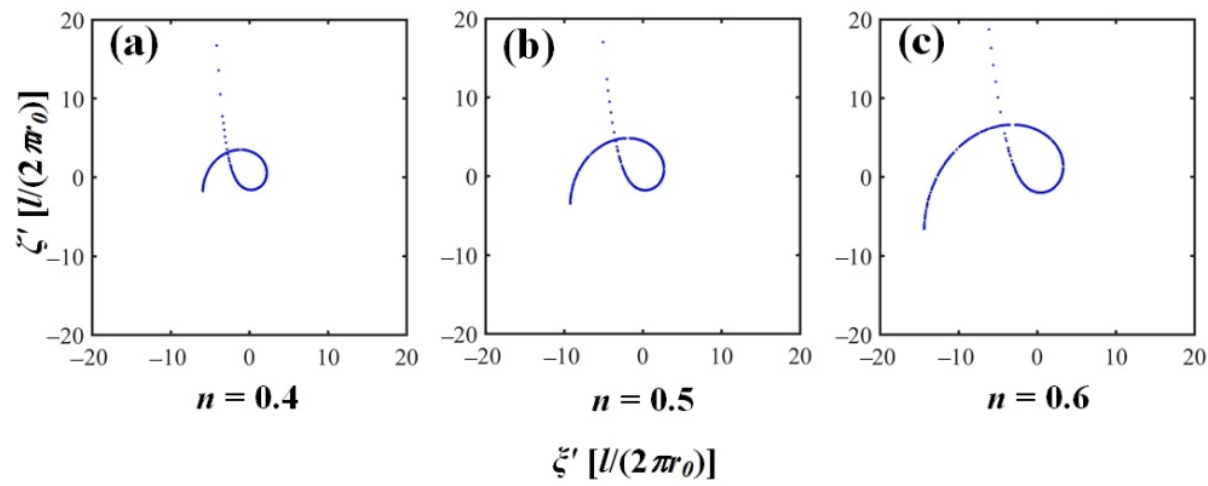

Figure 2. Spot diagram of the local spatial frequencies for $l=-5$, (a) $n=0.4$, (b) $n=0.5$ and (c) $n=0.6$.

The focal fields of the spoon-like beams can be better understood by analyzing the transverse energy flow of the beam [30]. Figure 3a-c show the transverse energy flow map of the spoon-like beams shown Figure 1d-f. The direction and magnitude of the arrows in Figure $3 \mathrm{a}-\mathrm{c}$ demonstrate the counterpart of the energy flow at the focal plane. The energy at the focal plane flows towards the head of the spoon-like beam. Furthermore, the energy around the head of the spoon-like beams when $n=0.4,0.5$, and 0.6 exhibits a stronger and larger density distribution. There is an intensity gradient along the spoon-like trajectory of the beam. The gradient force is proportional to the intensity gradient and points in the direction of the intensity gradient (toward the head of the spoon-like beam) [31]. Thus, the gradient force can drive microparticles move toward the head of the beam.
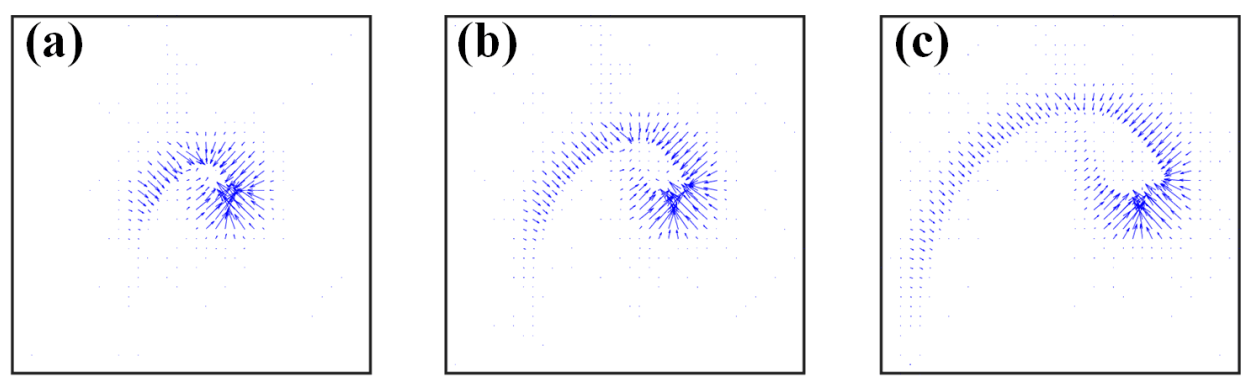

Figure 3. $(\mathbf{a}-\mathbf{c})$ The transverse energy flow of the spoon-like beams with $l=-5, n=0.4,0.5$, and 0.6 at the focal plane, respectively. 
The proposed spoon-like beams can be customized with the tailored exponential phase. The tailored exponential phase can be written as:

$$
\begin{aligned}
& \psi(r, \theta)=\left\{\begin{array}{lc}
\psi(r, \theta) & p \cdot \min [\psi(r, \theta)] \leq \psi(r, \theta) \leq 0 \\
0 & \text { else }
\end{array} \quad(l<0)\right.
\end{aligned}
$$

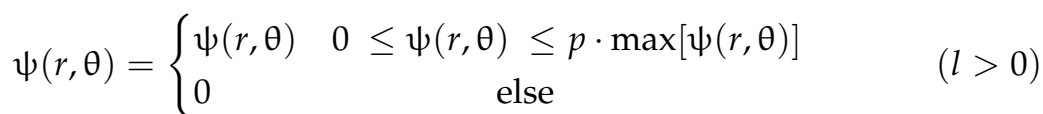

We take the exponential phase shown in Figure 1c as an example. The bored phase profiles with $l=-5$ and $n=0.6$ can be calculated with Equation (4). Figure $4 \mathrm{a}-\mathrm{d}$ show the calculated phases with the parameters $p=1,0.05,0.1$ and 0.15 , respectively. The simulated focal fields of the customized spoon-like beams generated with the tailored phases in Figure $4 \mathrm{a}-\mathrm{d}$ are shown in Figure $4 \mathrm{e}-\mathrm{h}$, respectively. The results demonstrate that the intensity trajectory of the spoon-like beam can be customized by adjusting the exponential phase with Equation (4) or (5).
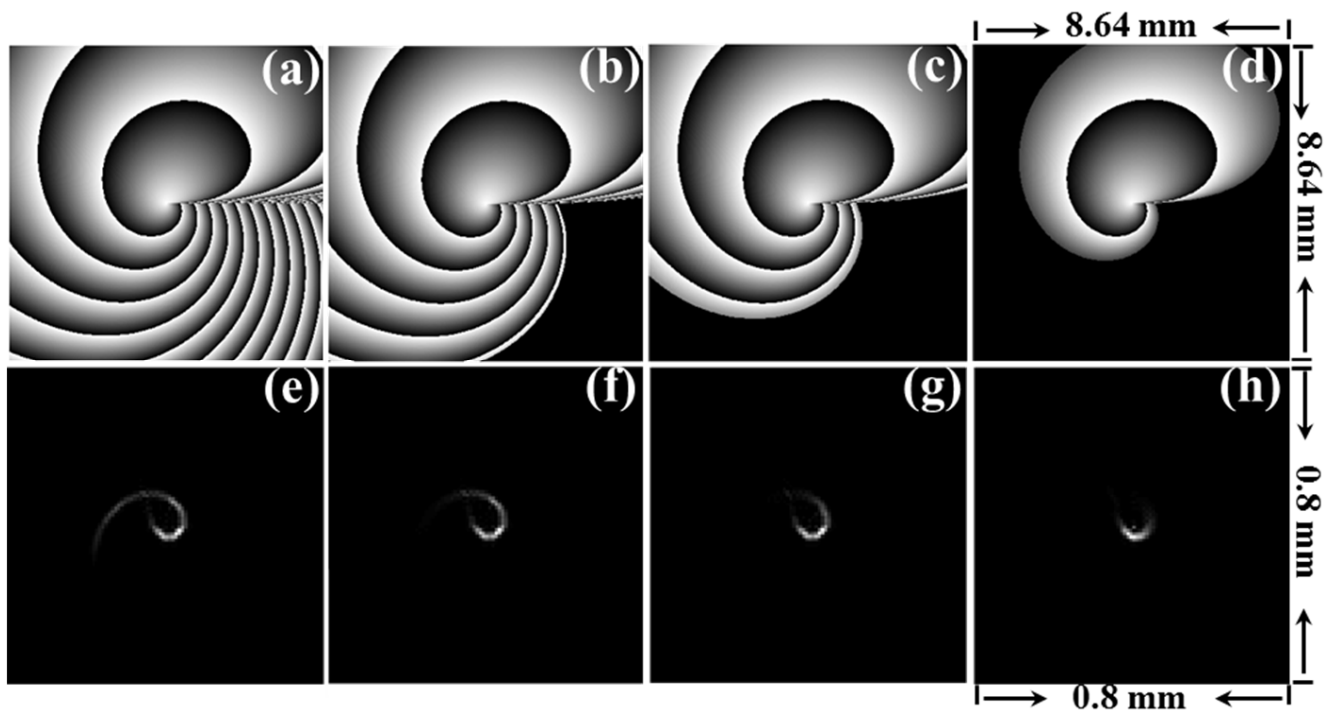

Figure 4. (a-d) The tailored exponential phases with $l=-5, n=0.6$, and $p=1,0.05,0.1$ and 0.15, respectively. $(\mathbf{e}-\mathbf{h})$ Simulated intensity patterns of the customized spoon-like beams generated with the tailored phases in (a-d), respectively.

\section{Experiments and Results}

In this paper, we experimentally verify the two-dimensional intensity patterns of the spoon-like beams at the focal plane of a Fourier transforming lens. Furthermore, we also demonstrate the optical guiding of microparticles with the spoon-like beam. The optical tweezers system shown in Figure 5 consists of an optically pumped semiconductor laser (Coherent Genesis, $1 \mathrm{~W}$, with a wavelength of $532 \mathrm{~nm}$ and adjustable laser power, Santa Clara, CA, USA), a beam expanding system with the focal lengths of the lens ( $L_{1}$ and $L_{2}$ : $f_{1}=30 \mathrm{~mm}$ and $f_{2}=300 \mathrm{~mm}$ ), a spatial light modulator (SLM, Holoeye, Pluto-VIS-096, $1920 \times 1080$ pixels, $8 \mu \mathrm{m} \times 8 \mu \mathrm{m} /$ pixel, reflective type, Berlin, Germany), an inverted telescope system $\left(L_{3}\right.$ and $L_{4}: f_{3}=300 \mathrm{~mm}$ and $\left.f_{4}=100 \mathrm{~mm}\right)$, and an inverted microscope with a $100 \times$ Olympus (Tokyo, Japan) oil-immersion objective (N.A. 1.3).

In the experimental setup shown in Figure 5, a CCD camera (Beijing, China) can be inserted in the focal plane of the lens $L_{3}$ to capture the intensity patterns of the desired beams generated with the exponential phases shown in Figures $1 \mathrm{a}-\mathrm{c}$ and $4 \mathrm{a}-\mathrm{d}$. Figure $6 \mathrm{a}-\mathrm{c}$ present the CCD-captured intensity patterns of the spoon-like beams with $n=0.4,0.5$ and 0.6 in Figure 1d-f, respectively. It can be seen in Figure $6 a-c$ that the CCD-captured intensity patterns agree with the spot diagram of the local spatial frequencies demonstrated in Figure $2 \mathrm{a}-\mathrm{c}$, respectively. Figure $7 \mathrm{a}-\mathrm{d}$ show the CCD-captured intensity patterns of 
the customized spoon-like beams shown in Figure 4e-h. The experimental results were consistent with the simulated ones.

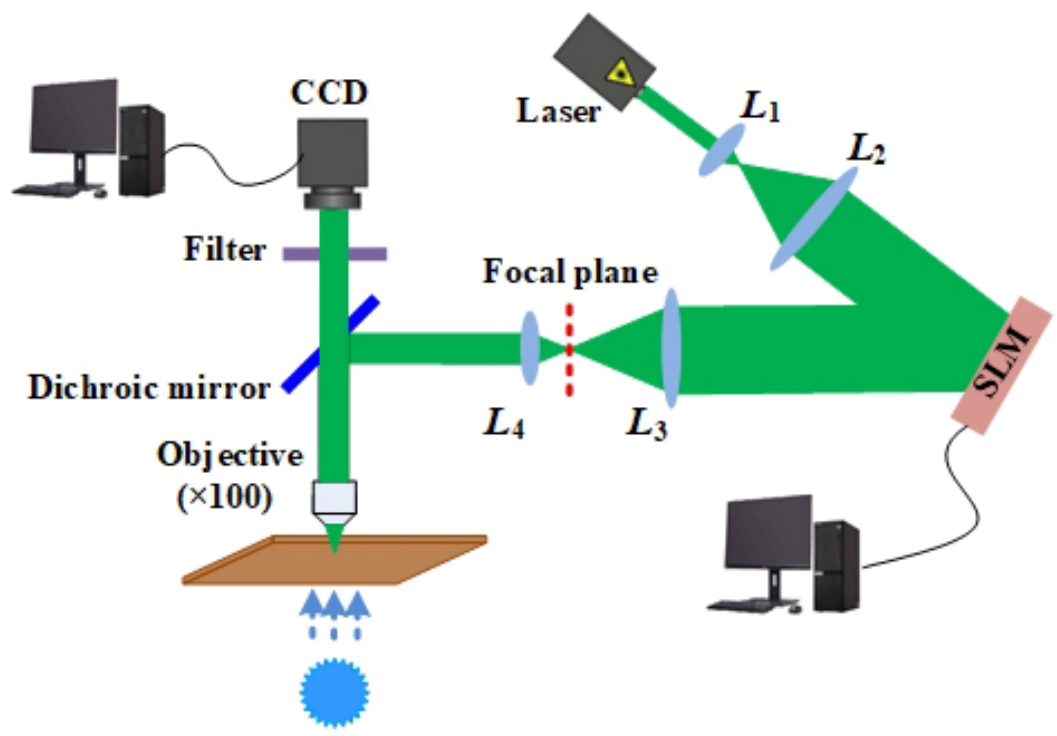

Figure 5. Schematic of the experimental setup.
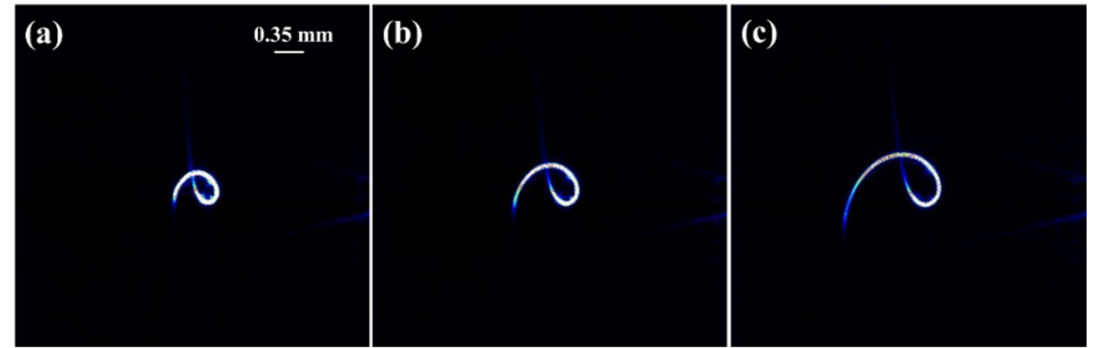

Figure 6. (a-c) CCD-captured intensity patterns of the spoon-like beams with $l=-5, n=0.4,0.5$ and 0.6 , respectively.
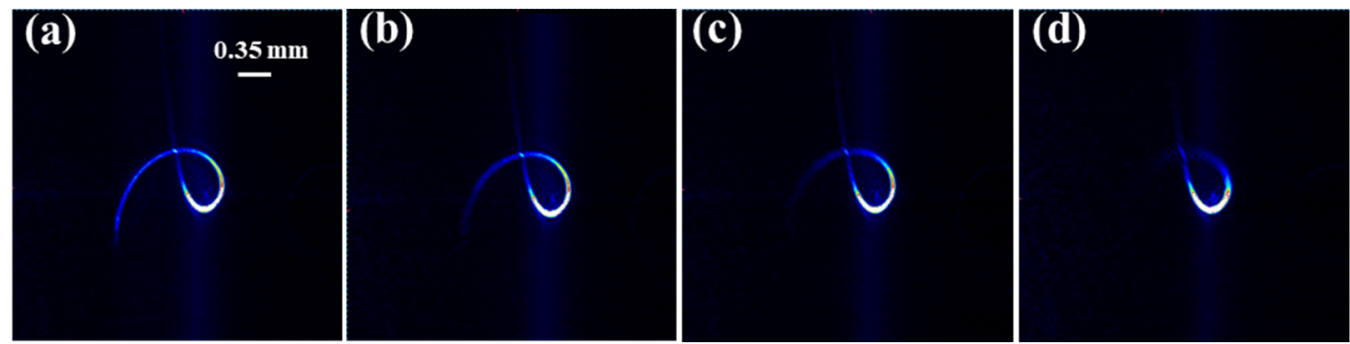

Figure 7. (a-d) CCD-captured intensity patterns of the customized spoon-like beams generated with the phase profiles in Figure $4 a-d$, respectively.

As an example, the spoon-like beam with $l=-5$ and $n=0.4$ was used to manipulate microparticles in the tweezers system. Silica beads with a diameter of about $4 \mu \mathrm{m}$ were suspended in the deionized water of with refractive index of 1.33 and used as the manipulated objects. The corresponding phase profile is shown in Figure 8a. When the phase profile was loaded on the SLM, the corresponding spoon-like beam was reconstructed at the focal plane of the objective, which is shown in Figure $8 \mathrm{~b}$. As we known, the intensity-gradient force exerted on microparticles is attributed to the intensity gradient of an optical beam [31]. When the output power was set as $0.6 \mathrm{~W}$, we can observe that microparticles were attracted to the trap by the intensity-gradient force, and then driven along the spoon-like route. Figure $8 \mathrm{c}-\mathrm{f}$ show the optical guiding of microparticles automatically along the spoon-like 
trajectory. The white-dashed lines in Figure 8c-f annotate the approximate regions of the generated spoon-like beams, the red arrow shown in Figure 8c shows the direction of the optical guiding (the direction of intensity gradient), and the microparticle is marked with a black arrow. Such spoon-like beams can realize the optical guiding of microparticles automatically along the intensity trajectory. The experimental results agreed with the ones shown in Figure 3a-c.

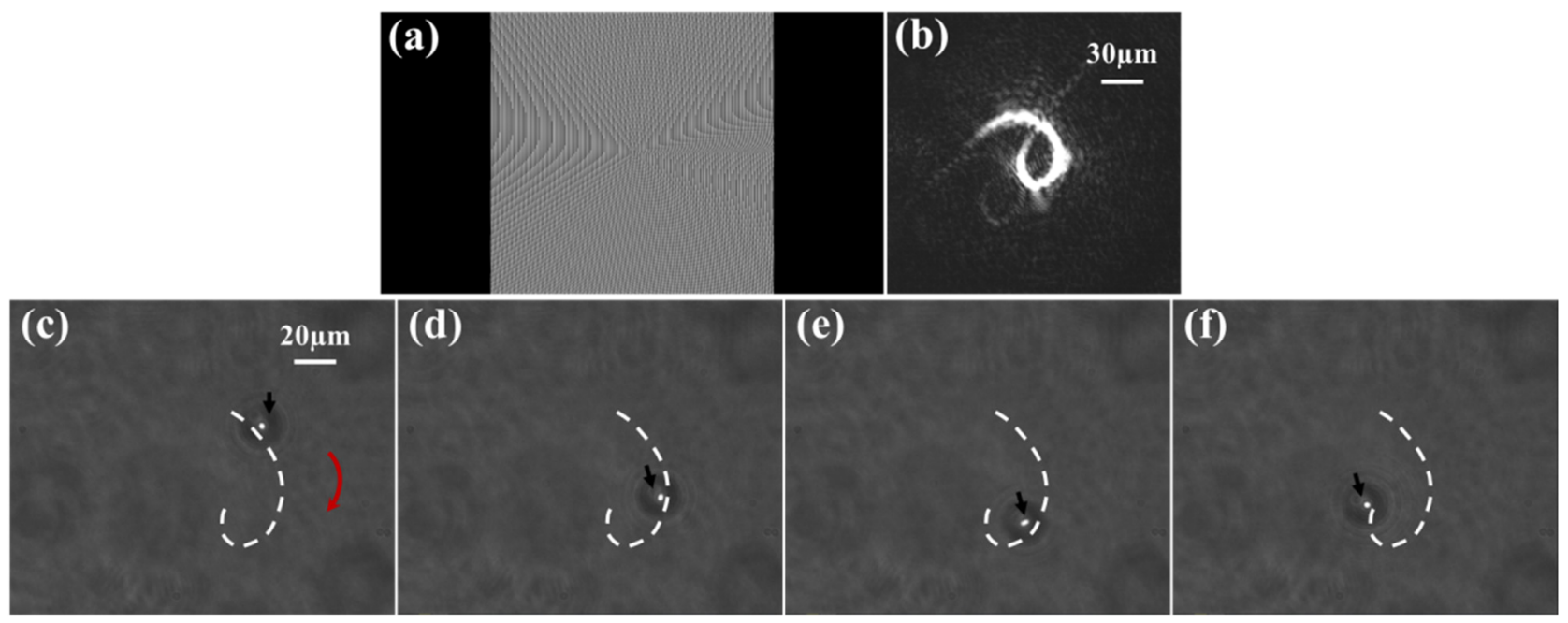

Figure 8. (a) The phase profiles used to generate the spoon-like beams with $l=-5, n=0.4$. (b) The reconstructed spoon-like beam at the focal plane of the objective. (c-f) The trapped microspheres moving automatically along the spoon-like trajectory of the generated beam with $l=-5, n=0.4$.

\section{Conclusions}

A spoon-like beam was generated with an exponential phase in this paper. The intensity distributions of the beams at the focal plane were analyzed theoretically and experimentally. The result demonstrates that the intensity trajectory of the spoon-like beam can be customized by the tailored exponential phases with different parameters, p. It was also proven that the proposed spoon-like beam can manipulate microparticles automatically. The beam has potential application in the field of optical trapping.

Author Contributions: Conceptualization, L.G., Y.T., T.X. and S.C.; data curation, Y.X. (Yiping Xu), Y.X. (Yan Xiong), X.L. and S.T.; formal analysis, Y.T. and Y.X. (Yiping Xu); methodology, L.G. and T.X.; resources, S.T.; software, L.G. and T.X.; data curation, L.G. and T.X.; writing-original draft preparation, L.G. and T.X.; writing-review and editing, S.C. and Y.T. All authors have read and agreed to the published version of the manuscript.

Funding: National Natural Science Foundation of China (NSFC) (Grant Nos. 11904032, 52004087); the Science Foundation of Educational Commission of Hubei Province of China (Grant No. T2020008).

Institutional Review Board Statement: Not applicable.

Informed Consent Statement: Not applicable.

Data Availability Statement: Publicly available datasets were analyzed in this study. This data can be found here: [https://www.lumerical.com/] (accessed on 30 December 2021).

Conflicts of Interest: The authors declare no conflict of interest.

\section{References}

1. Ashkin, A. Forces of a single-beam gradient laser trap on a dielectric sphere in the ray optics regime. J. Biophys. 1992, 61, 569-582. [CrossRef]

2. Liu, X.; Zhao, D. Optical trapping Rayleigh particles by using focused multi-Gaussian Schell-model beams. Appl. Opt. 2014, 53, 3976-3981. [CrossRef] [PubMed] 
3. Shen, Y.; Wang, X.; Xie, Z.; Min, C.; Fu, X.; Liu, Q.; Yuan, X. Optical vortices 30 years on: OAM manipulation from topological charge to multiple singularities. Light Sci. Appl. 2019, 8, 1-29. [CrossRef] [PubMed]

4. Zhang, H.; Li, J.; Guo, M.; Duan, M.; Feng, Z.; Yang, W. Optical trapping two types of particles using a focused vortex beam. Optik 2018, 166, 138-146. [CrossRef]

5. Zhang, Z.; Cannan, D.; Liu, J.; Zhang, P.; Christodoulides, D.N.; Chen, Z. Observation of trapping and transporting airborne absorbing particles with a single optical beam. Opt. Express 2012, 20, 16212-16217. [CrossRef]

6. Shvedov, V.G.; Desyatnikov, A.S.; Rode, A.V.; Izdebskaya, Y.V.; Krolikowski, W.Z.; Kivshar, Y.S. Optical vortex beams for trapping and transport of particles in air. Appl. Phys. A 2010, 100, 327-331. [CrossRef]

7. Tao, S.; Yuan, X.; Lin, J.; Peng, X.; Niu, H. Fractional optical vortex beam induced rotation of particles. Opt. Express 2003, 13, 7726-7731. [CrossRef]

8. Zeng, J.; Liang, C.; Wang, H.; Wang, F.; Zhao, C.; Gbur, G.; Cai, Y. Partially coherent radially polarized fractional vortex beam Opt. Express 2020, 28, 11493-11513. [CrossRef]

9. Lin, J.; Yuan, X.; Tao, S.; Peng, X.; Niu, H. Deterministic approach to the generation of modified helical beams for optical manipulation. Opt. Express 2005, 13, 3862-3867. [CrossRef]

10. Li, P.; Liu, S.; Peng, T.; Xie, G.; Gan, X.; Zhao, J. Spiral autofocusing airy beams carrying power-exponent-phase vortices. Opt. Express 2014, 22, 7598-7606. [CrossRef]

11. Li, J.; Sun, P.; Ma, H.; Zhou, S. Focus properties of cosh-Gaussian beams with the power-exponent-phase vortex. J. Opt. Soc. Am. A 2020, 37, 483-490. [CrossRef] [PubMed]

12. Zeng, J.; Zhang, Q.; Liu, X.; Chen, Y.; Xu, Y.; Jin, Y.; Xiong, Y.; Tao, S.; Yang, W.; Cheng, S. Autofocusing Airy beams carrying a new kind of power-exponent-phase vortices. Opt. Commun. 2022, 507, 127635. [CrossRef]

13. Garcés-Chávez, V.; McGloin, D.; Melville, H.; Sibbett, W.; Dholakia, K. Simultaneous micromanipulation in multiple planes using a self-reconstructing light beam. Nature 2002, 419, 145-147. [CrossRef] [PubMed]

14. Durnin, J.; Miceli, J.J., Jr.; Eberly, J.H. Diffraction-free beams. Phys. Rev. Lett. 1987, 58, 1499-1501. [CrossRef]

15. Liu, Z.; Tang, X.; Zhang, Y.; Zhang, Y.; Ma, L.; Zhang, M.; Yuan, L. Simultaneous trapping of low-index and high-index microparticles using a single optical fiber Bessel beam. Opt. Lasers Eng. 2020, 131, 106119. [CrossRef]

16. Tao, S.; Yuan, X. Self-reconstruction property of fractional Bessel beams. J. Opt. Soc. Am. A 2004, 21, 1192-1197. [CrossRef]

17. Alonzo, C.A.; Rodrigo, P.J.; Glückstad, J. Helico-conical optical beams: A product of helical and conical phase fronts. Opt. Express 2005, 13, 1749-1760. [CrossRef]

18. Wu, H.; Zhang, F.; Zhang, Z. Droplet breakup and coalescence of an internal-mixing twin-fluid spray. Phys. Fluids 2021, 33, 013317. [CrossRef]

19. Xie, Y.; Meng, X.; Wang, F.; Jiang, Y.; Ma, X.; Wan, L.; Huang, Y. Insight on corrosion behavior of friction stir welded AA2219/AA2195 joints in astronautical engineering. Corros. Sci. 2021, 192, 109800. [CrossRef]

20. Zhang, Q.; Lin, W.; Wang, Y.; Wu, X.; Luo, Y. Control strategy of the vehicle's active steer by wire. J. Coast. Res. 2020, 103, 355-360. [CrossRef]

21. Zhang, W.; Zhang, Z. Deck electrical system support technology of port tanker. J. Coast. Res. 2020, 103, 392-396. [CrossRef]

22. Baluyot, S.; Hermosa, N. Intensity profiles and propagation of optical beams with bored helical phase. Opt. Express 2009, 17, 16244-16254. [CrossRef] [PubMed]

23. Lao, G.; Zhang, Z.; Zhao, D. Propagation of the power-exponent-phase vortex beam in paraxial ABCD system. Opt. Express 2016, 24, 18082-18094. [CrossRef] [PubMed]

24. Shen, D.; Wang, K.; Zhao, D. Generation and propagation of a new kind of power-exponent-phase vortex beam. Opt. Express 2019, 27, 24642-24653. [CrossRef] [PubMed]

25. Xia, T.; Tao, S.; Cheng, S. A spiral-like curve with an adjustable opening generated by a modified helico-conical beam. Opt. Commun. 2019, 18, 105603. [CrossRef]

26. Cheng, S.; Xia, T.; Liu, M.; Jin, Y.; Zhang, G.; Xiong, Y.; Tao, S. Power-exponent Helico-conical optical beams. Opt. Laser Technol. 2019, 117, 288-292. [CrossRef]

27. Cederquist, J.; Tai, A.M. Computer-generated holograms for geometric transformations. Appl. Opt. 1984, 23, 3099-3104. [CrossRef]

28. Bryngdahl, O. Optical map transformations. Opt. Commun. 1974, 10, 164-168. [CrossRef]

29. Bryngdahl, O. Geometrical transformations in optics. J. Opt. Soc. Am. 1974, 64, 1092. [CrossRef]

30. Sztul, H.I.; Alfano, R.R. The Poynting vector and angular momentum of airy beams. Opt. Express 2008, 16, 9411-9416. [CrossRef]

31. Ashkin, A.; Dziedzic, J.M.; Bjorkholm, J.E.; Chu, S. Observation of a single-beam gradient force optical trap for dielectric particles. Opt. Lett. 1986, 11, 288-290. [CrossRef] [PubMed] 